\title{
The theory of pulsar winds and nebulæ
}

\author{
J.G. Kirk ${ }^{1}$, Y. Lyubarsky ${ }^{2}$, and J. Pétri ${ }^{1}$ \\ 1 Max-Planck-Insititut für Kernphysik, Postfach 1039 80, 69029 Heidelberg, \\ Germany John.Kirk@mpi-hd.mpg.de, Jerome.Petri@mpi-hd.mpg.de \\ 2 Department of Physics, Ben Gurion University, P.O.Box 653, Beer-Sheva 84105, \\ Israel lyub@bgu.ac.il
}

Summary. We review current theoretical ideas on pulsar winds and their surrounding nebulæ. Relativistic MHD models of the wind of the aligned rotator, and of the striped wind, together with models of magnetic dissipation are discussed. It is shown that the observational signature of this dissipation is likely to be point-like, rather than extended, and that pulsed emission may be produced. The possible pulse shapes and polarisation properties are described. Particle acceleration at the termination shock of the wind is discussed, and it is argued that two distinct mechanisms must be operating, with the first-order Fermi mechanism producing the high-energy electrons (above $1 \mathrm{TeV}$ ) and either magnetic annihilation or resonant absorption of ion cyclotron waves responsible for the $100 \mathrm{MeV}$ to $1 \mathrm{TeV}$ electrons. Finally, MHD models of the morphology of the nebula are discussed and compared with observation.

\section{Introduction}

The theory of pulsar winds and the nebulæ they energise is currently celebrating its golden jubilee. Ten years before the discovery of pulsars it was already apparent that the magnetic field and relativistic particles that produce the radiation of the Crab Nebula must have their origin in a central stellar object (Piddington, 1957). Today, about 50 similarly powered objects are known, and some of them, like the Crab, are detected and even resolved at all accessible photon frequencies, from the radio to $\mathrm{TeV}$ gamma-rays. The rotation of the central neutron star (Pacini, 1967) is now universally accepted as the energy source fuelling these objects, but the details of the coupling mechanism are still unclear. In this article we review current theoretical ideas on this subject and their relationship to observations. We concentrate on the magnetohydrodynamic description of the relativistic outflow driven by the pulsar and on the bubble it inflates in the surrounding medium.

The discussion is organised as follows: in section 2 we consider the region between the surface of the neutron star and the light cylinder a surface of cylindrical radius $r_{\mathrm{L}}=c P /(2 \pi)$, where $P$ is the pulsar period. The speed of an object that corotates with the star becomes luminal on this surface, and 
the wavelength of the radiation that would be emitted by the pulsar in vacuum is $2 \pi r_{\mathrm{L}}$. In the terminology of radiating systems, the region within the light cylinder is, therefore, the "near zone", where the fields can be approximated as being in rigid corotation. Conventionally, this region is called the pulsar magnetosphere. It is thought to be the site of copious pair creation, and, in most theories, is the region in which the pulsed radiation itself is emitted.

Electric and magnetic fields dominate the dynamics in the near zone inside the light cylinder. However, this is also true in at least part of the "far zone", that lies well outside the light cylinder - a region that is not conventionally thought of as the pulsar magnetosphere. Here, we follow conventional terminology, and call this the wind zone $e^{3}$; it extends up to the termination shock, where the ordered, relativistic flow of particles and fields is randomised. Electromagnetic fields dominate the inner part of the wind zone, where the plasma flows subsonically with respect to the fast magnetosonic mode. As we describe in section 3 , force-free solutions to the aligned rotator problem show that the plasma accelerates up to the critical point where its velocity equals the fast magnetosonic speed, and, presumably, passes through it into a supersonic domain. In this part of the flow, the dynamics is strongly influenced by the particles, although most of the energy flux continues to be carried by the fields. The oblique rotator is a more suitable model for a pulsar, and the key difference it introduces in the wind zone is wave-like structure on the very small length scale $r_{\mathrm{L}}$. We discuss the structure of such a striped wind in section 4 , and consider the possibility that dissipative processes play a role in converting Poynting flux into kinetic energy. In both the sub and supersonic parts, the pulsar wind is highly relativistic. If it radiates at all, its emission is beamed predominantly in the radial direction. This simple kinematic effect has an important influence on the radiative signature of this region, as we discuss in section 5 .

Observationally, the termination shock can be identified as the outer boundary of an underluminous region that lies at the centre of the diffuse synchrotron emission of the nebula. The nebula itself, therefore, lies between the termination shock and an outer boundary, where the relativistic particles produced by the pulsar impinge on either the debris of its natal supernova explosion, or on the interstellar medium. These particles are most plausibly accelerated at the termination shock, and we consider the acceleration processes operating here in section 6 . Finally, in section 7 , we discuss models of the nebula and its emission.

\footnotetext{
${ }^{3}$ Note that we designate the entire far zone outside the light cylinder as the pulsar wind, although this term is really only appropriate for the supersonic part of the flow.
} 


\section{The magnetosphere}

An enormous electric field is induced by the rotation of a magnetised neutron star. This field tears particles from the stellar surface and accelerates them up to high energies. As a result, these "primary" particles initiate an electronpositron cascade, which, according to conventional wisdom, populates the entire magnetosphere with plasma. In the aligned case, solutions have been found for the region well within the light cylinder in which this plasma is confined to domes above the poles and a differentially rotating equatorial disk (Krause-Polstorff \& Michel, 1985; Pétri et al., 2002). If, on the other hand, the magnetosphere is filled with plasma, the strength of the magnetic field is sufficient to ensure that the plasma corotates (for recent reviews, see Pétri, 2006; Harding \& Lai, 2006). At some point near the light cylinder, corotation must cease, and the particles escape, carrying away magnetic flux and energy in the form of an ultrarelativistic, magnetised wind.

If one traces the escaping ("open") magnetic field lines back to the stellar surface, they define areas which, in a dipole geometry, lie close to the magnetic axis and are called "polar caps". To maintain a steady state, plasma must be continuously generated on the open field lines in these polar regions. It then streams along them with relativistic velocity and eventually escapes through the light cylinder. It is usually assumed that the mechanism responsible for creating the pulsed radiation is somehow associated with the materialisation of these plasma streams, which presumably takes place at either an "inner gap" or "outer gap", (e.g., Harding, 2005). However, this is not necessarily true (see section 5), and, at least for the optical pulses, there are indications that the site of pulse production could lie outside the light cylinder (Kirk et al., 2002; Dyks et al., 2004; Pétri \& Kirk, 2005).

The rate at which pairs escape is conveniently measured in terms of the the pair multiplicity, $\kappa$, which is the number of pairs produced by a single primary particle that emerges from one of the polar caps. The primary beam consists of particles of a single charge, and its density is expected to be close to the Goldreich-Julian density, defined as that required to screen the induced electric field, namely

$$
n_{\mathrm{GJ}} \equiv|(\boldsymbol{\Omega} \cdot \mathbf{B}) /(2 \pi e c)|
$$

(e.g., Davis, 1947; Hones \& Bergeson, 1965; Michel, 1991) where $\Omega$ is the angular velocity of the star and $\mathbf{B}$ the magnetic field. In an aligned dipole geometry, the foot-points of those field lines that cross the light cylinder lie within polar caps of (cylindrical) radius $r_{\mathrm{pc}} \approx \sqrt{R_{*}^{3} / r_{\mathrm{L}}}$, where $R_{*}$ is the stellar radius, and this is a reasonable approximation also in the oblique case (e.g., Dyks et al., 2004). Assuming the pairs stream away from the polar cap at relativistic speed, the total number ejected per unit time may therefore be estimated as

$$
\dot{N}=\kappa \frac{\Omega^{2} B_{*} R_{*}^{3}}{e c}
$$




$$
=2.7 \times 10^{30} \kappa\left(\frac{B_{*}}{10^{12} \mathrm{G}}\right)\left(\frac{P}{1 \mathrm{~s}}\right)^{-2} \mathrm{~s}^{-1}
$$

where $\mathbf{B}_{*}$ is the magnetic field at the polar cap.

The actual value of the multiplicity, $\kappa$, is rather uncertain. Theoretical models (Hibschman \& Arons, 2001a,b), give values of $\kappa$ ranging from a few to thousands, but some observations suggest that the real value might be substantially higher (Gallant \& Tuffs, 2002; Bietenholz et al., 2004, see section 7). If $\boldsymbol{\Omega} \cdot \mathbf{B}_{*}<0$, the primary particles are positively charged ions. Because these particles do not breed in cascades, they make up a fraction of at most $1 / \kappa$ by number of the wind particles. Nevertheless, if $\kappa$ is indeed as low as predicted theoretically, the energy flux carried by ions could be as large as that carried by pairs.

\section{The wind of an aligned rotator}

Pairs are produced with an energy that ranges from dozens to hundreds of $\mathrm{MeV}$, and the total energy density of the produced plasma remains small compared to the magnetic energy density. Nevertheless, the presence of the plasma is crucially important, because the electromagnetic structure in the far zone is strongly influenced by the currents it carries. The overall structure may be pictured by taking into account that the magnetic field is frozen into the plasma. As the plasma cannot rotate with superluminal velocity, the magnetic field lines beyond the light cylinder are wrapped backwards with respect to the rotation of the neutron star. As a result of this, even an axisymmetric rotator loses energy by driving a plasma wind, provided its inner zone is filled with plasma. In contrast, an aligned magnetic dipole rotating in vacuum does not lose energy.

In the case of an aligned rotator (with parallel magnetic dipole and rotation axes), the entire system is axisymmetric. Essentially, the rotational energy of the neutron star is spent in the generation of azimuthal magnetic field in the wind. This can be seen by noting that the electric field $\left(\mathbf{E}^{\prime}\right)$ in the proper frame of the flow vanishes because the plasma conductivity is infinite, which implies

$$
\mathbf{E}+(1 / c) \mathbf{v} \times \mathbf{B}=0 .
$$

In a radial, relativistic wind, the poloidal component of $\mathbf{B}$ is also radial, so that the magnitude of the electric field is close to that of the azimuthal magnetic field, which then determines the radial component of the Poynting flux: $P_{R} \approx$ $c B_{\varphi}^{2}$.

In a steady, axisymmetric solution, the displacement current vanishes, and the azimuthal magnetic field is generated by poloidal currents that flow either into or out of the polar caps of the star, depending on the $\operatorname{sign}$ of $\boldsymbol{\Omega} \cdot \mathbf{B}_{*}$. The circuit is closed in a current carrying surface - a "current sheet". Well outside the light cylinder, this sheet lies in the equatorial plane and separates 
the field lines that originate from the two magnetic poles. Inside the light cylinder, the current flows in the surface that encloses the volume containing closed field lines.

At the light cylinder, the azimuthal and poloidal fields are comparable. In the wind zone, conservation of magnetic flux in a diverging flow implies that the poloidal field decreases faster than the azimuthal field, which is proportional to $1 / r$, where $r$ is the cylindrical radius. Thus, the field in the far zone of the wind may be considered as purely azimuthal; even though each magnetic field line is a spiral anchored on the surface of the star, the spiral becomes so tightly wound in the far zone that it can be approximated locally as separate coaxial magnetic loops moving together with the flow.

The relative strength of magnetic field and particles is an important characteristic of the wind. This is best defined, in an ideal MHD description, as the ratio in the proper frame (where $\mathbf{E}^{\prime}=0$ ) of the magnetic to particle enthalpy densities:

$$
\sigma \equiv \frac{B^{\prime 2}}{4 \pi w c^{2}}
$$

where $w$ is the proper enthalpy density of the plasma and $\mathbf{B}^{\prime}$ is the magnetic field in the proper frame. If, as we assume, the plasma is cold, the enthalpy density is simply the rest mass energy density: $w=m_{\mathrm{e}} c^{2} n / \Gamma$, where $n$ is the number density in the lab. frame, (in which the centre of mass of the neutron star is at rest) and $\Gamma$ is the Lorentz factor of the wind in this frame. Then, since the velocity of the wind is perpendicular to $\mathbf{B}^{\prime}=\mathbf{B} / \Gamma, \sigma$ equals the ratio of the energy carried by Poynting flux to that carried by particles:

$$
\sigma=B^{2} / 4 \pi m_{\mathrm{e}} c^{2} n \Gamma
$$

According to all available models of the multiplicity, $\sigma \gg 1$ close to the light cylinder, so that the wind is Poynting dominated at that point. This means that the fast magnetosonic speed $v_{\text {fms }}$ is very close to $c$ :

$$
\begin{aligned}
v_{\mathrm{fms}} & =\frac{c B^{\prime}}{\sqrt{4 \pi w+B^{\prime 2}}} \\
& \approx \sqrt{\frac{\sigma}{1+\sigma}}
\end{aligned}
$$

(e.g., Kirk \& Duffy, 1999) and the corresponding Lorentz factor is $\Gamma_{\mathrm{fms}}=\sqrt{\sigma}$. In the cold wind, $\sigma$ is related to $\Gamma$ via Eq. (5), so that the fast magnetosonic point is located at the point where

$$
\begin{aligned}
\Gamma & =\Gamma_{\mathrm{fms}}=\left(\frac{B^{2}}{4 \pi m_{\mathrm{e}} c^{2} n}\right)^{1 / 3} \\
& \equiv \mu_{\mathrm{M}}^{1 / 3}
\end{aligned}
$$

where $\mu_{\mathrm{M}}$ is the magnetisation parameter introduced by Michel (1969). The propagation of the fast magnetosonic wave is a result of the interplay between 
magnetic tension and plasma inertia. If, as seems likely, the flow emerges through the light cylinder subsonically, i.e., with $\Gamma \ll \sqrt{\sigma}$, the dynamics simplify significantly, because the inertia terms in the equation of motion are unimportant. Since gravity and gas pressure are also unimportant in the pulsar case, this "force-free" approximation implies that the Lorentz force is exactly cancelled by the electric force:

$$
\rho_{e} \mathbf{E}+(1 / c) \mathbf{j} \times \mathbf{B}=0
$$

The description of the dynamics is complete when this equation is complemented by Maxwell's equations, connecting the charge and current densities $\rho_{e}$ and $\mathbf{j}$ with the fields $\mathbf{E}$ and $\mathbf{B}$, and by the ideal MHD condition (3).

The force-free MHD equations for the pulsar magnetosphere are strongly nonlinear and must, in general, be solved numerically (Contopoulos et al., 1999; Gruzinov, 2005; Komissarov, 2006; McKinney, 2006; Timokhin, 2006). In particular, the oblique rotator, being a three-dimensional problem, has only recently been treated (Spitkovsky, 2006). Nevertheless, an exact axisymmetric solution describing a magnetosphere of a rotating star, namely that of the split monopole, has been known for many years (Michel, 1973). In this solution, magnetic field lines extend from the origin to infinity in the upper hemisphere and converge from infinity to the origin in the lower hemisphere. The hemispheres are separated by an equatorial current sheet. The magnetic surfaces have the form of coaxial cones whose vertices lie at the origin. Plasma flows radially from the origin to infinity. Of course, the flow lines in a realistic (dipole) magnetosphere cannot be radial everywhere; there should be a zone of closed field lines inside the light cylinder. However, Ingraham (1973) and Michel (1974) showed that, independently of the field structure near the origin, the flow lines beyond the light cylinder become asymptotically radial in the force-free approximation, just as in the split monopole solution. This is in contrast with the situation in axisymmetric nonrelativistic MHD winds where hoop stresses collimate the flow along the rotation axis. The reason is that in the relativistic case, the electric force compensates these stresses almost exactly.

In axisymmetric MHD, the flow lines lie in magnetic surfaces and the electric field is perpendicular to these surfaces, which are, therefore, equipotentials. The force-free condition (9) implies that the currents also flow along the magnetic surfaces. Taking this into account, one can find the asymptotic behaviour of the basic quantities in the radial wind:

$$
\begin{array}{ll}
B_{\varphi} \propto r_{\mathrm{L}} / R & E \propto r_{\mathrm{L}} / R \\
B_{R} \propto r_{\mathrm{L}}^{2} / R^{2} & n \propto r_{\mathrm{L}}^{2} / R^{2}
\end{array}
$$

where $R$ is the radius in spherical polar coordinates. It follows from the higher order terms in the asymptotic solutions presented by Ingraham (1973) and Michel (1974) that the difference between $E$ and $B$ decreases with radius and the flow velocity approaches closer and closer to $c$, i.e., it accelerates. Buckley 
(1977) showed that, in the force-free approximation, the Lorentz factor of the flow grows linearly with the radius (see also Contopoulos \& Kazanas, 2002). Eventually, the Lorentz factor becomes comparable to that of the fast magnetosonic mode $\Gamma_{\mathrm{fms}}=\sqrt{\sigma}$. The plasma inertia becomes important at this point, and the force-free approximation breaks down.

If the pulsar wind is launched with $\sigma_{0} \gg 1$ and $\Gamma_{0} \ll \sqrt{\sigma_{0}}$, it is initially subsonic, the force-free approximation holds, and the flow accelerates. As $\Gamma$ increases, $\sigma$ decreases, since, in a radial flow with purely toroidal field, $B^{2} / n$ remains constant and $\sigma \propto \Gamma^{-1}$ according to eq. (5). At the fast magnetosonic point, $\sigma=\left(\sigma_{0} \Gamma_{0}\right)^{2 / 3} \gg 1$, and the flow is still Poynting dominated. This is in contrast with the nonrelativistic situation, where $v_{\mathrm{fms}}=B / \sqrt{4 \pi \rho}$ so that the energy flux carried by particles equals the Poynting flux at the fast magnetosonic point. The reason is that in the relativistic case, the electric and magnetic forces almost compensate each other, allowing inertial effects to come into play at an early stage.

Beyond the fast magnetosonic point the full relativistic MHD equations must be solved. However, the flow remains nearly radial even there (Tomimatsu, 1994; Beskin et al., 1998; Chiueh et al., 1998; Bogovalov \& Tsinganos, 1999; Lyubarsky \& Eichler, 2001), because the additional inertial forces tend to resist collimation. Thus, if the flow is radial in the force-free region, it remains radial further out. In fact, the flow is practically ballistic, i.e., the plasma moves radially with negligible acceleration, so that $\Gamma$ and, hence, $\sigma$ remain constant. Thus, within the scope of ideal MHD, the wind can be accelerated to at most Lorentz factors of a few times $\Gamma_{\mathrm{fms}}$, at which point $\sigma$ is still large. Beyond this point, electromagnetic energy is not transferred to the plasma. ${ }^{4}$

\footnotetext{
${ }^{4}$ This does not mean that $\sigma$ remains large in any Poynting-dominated flow. The relativistic MHD equations do not forbid acceleration of the flow and conversion of Poynting flux into kinetic energy. There exist many solutions that demonstrate explicitly the reduction of Poynting flux to the equipartition level or even below (Li et al., 1992; Vlahakis, 2004; Beskin \& Nokhrina, 2006). However this can happen only if the flow lines are not radial. In the above-mentioned solutions, this is achieved by a special choice of the poloidal flux distribution such that the total poloidal flux is infinite and the magnetic surfaces do not converge to origin. (Note that although the poloidal field is small beyond the light cylinder, the stress of this field may be not negligible in some cases because the hoop stress is nearly compensated by the electric force.) Whereas it is quite plausible that such solutions could be matched to boundary conditions above a disk, it is difficult to imagine that they could be made compatible with a star (effectively a point source) threaded by a finite magnetic flux. For this reason, we do not discuss them further here.
} 


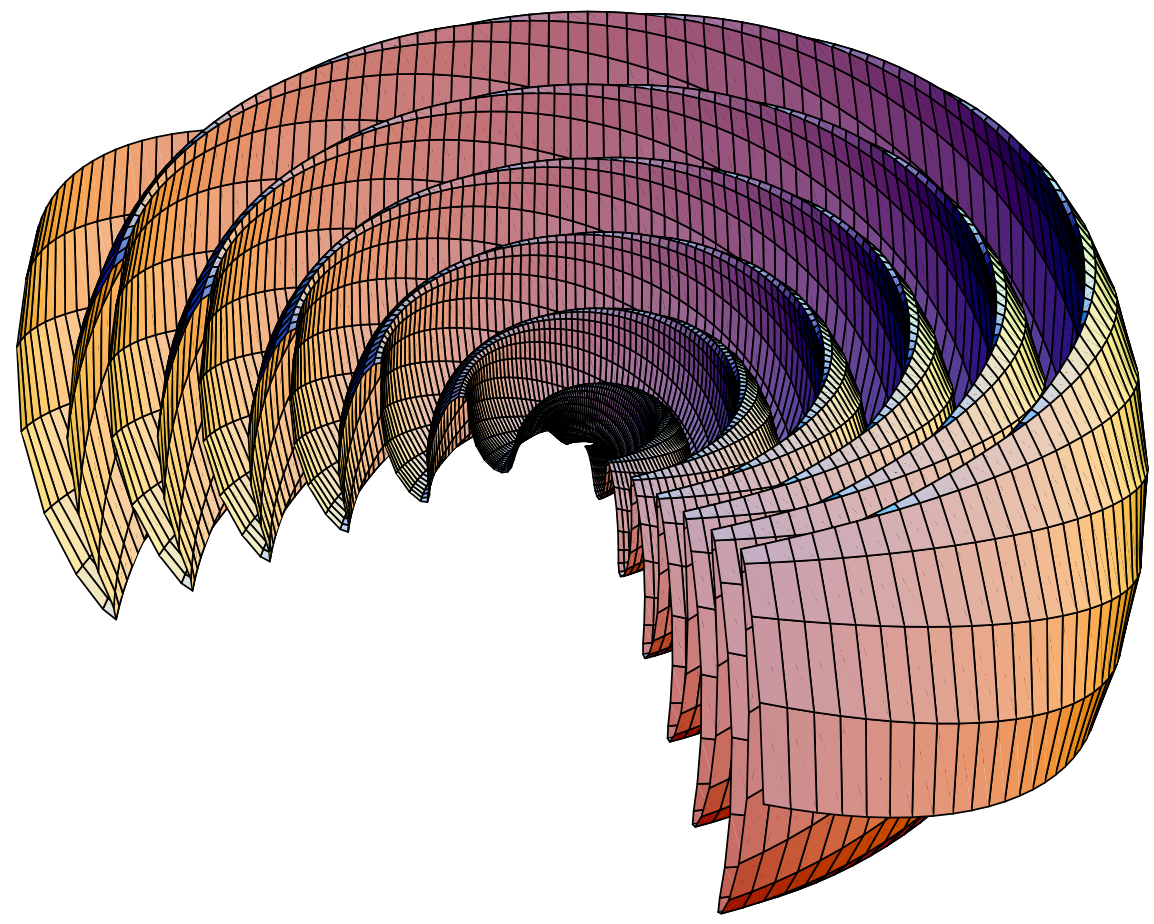

Fig. 1. The striped wind (Michel, 1971; Coroniti, 1990). A snapshot is shown of the surface that is traced out by the inclined magnetic equator when it rotates and is carried outwards by a radially propagating wind of constant velocity. This surface corresponds precisely to the current sheet in the oblique split-monopole solution (Bogovalov, 1999).

\section{The striped wind}

In the real world, pulsar winds are neither axisymmetric nor steady. In fact, the defining characteristic of a pulsar is that it is an oblique rotator, in the sense that its magnetic and rotation axes are not parallel. The time-varying electromagnetic fields excited by such an oblique rotator propagate outwards in the form of electromagnetic waves. The wavelength of these oscillations is at most $2 \pi r_{\mathrm{L}}$. In the far zone, this is much smaller than the radius. For this reason, dissipative processes, which operate only on short length scales, are likely to be much more important in the wind of an oblique rotator than in a steady, axisymmetric wind. Therefore, the dissipation or damping of these waves could be an important mechanism of energy transformation in a pulsar wind.

A rarefied magnetised plasma supports a variety of electromagnetic waves. However, it seems reasonable to simplify the problem by assuming that only MHD waves (i.e., those satisfying the ideal MHD condition (3)) survive in the 
far zone. The reason is that, according to the conventional picture, the plasma density at the base of the pulsar wind (at $r \sim r_{\mathrm{L}}$ ) is sufficiently large that low-frequency electromagnetic waves are heavily damped (e.g., Asseo et al., 1978; Melatos \& Melrose, 1996). In this case, it is once again useful to think of magnetic field lines as frozen into the plasma flow. In the equatorial belt, the magnetic field at a fixed radius alternates in direction at the frequency of rotation, being connected to a different magnetic pole every half-period. Michel (1971) pointed out that the flow in this zone should evolve into regions of cold, magnetically dominated plasma separated by very narrow, hot current sheets. Such a structure can be thought of as containing four tangential discontinuities per wavelength, each of which separates field lines from one magnetic pole from the thin, bounding layer of hot plasma which constitutes the current sheet. The corresponding structure in hydrodynamics is called an entropy wave. It is simply a stationary pattern of spatially varying temperature and density that is in pressure equilibrium in a motionless plasma.

The global picture of the resulting flow is shown in figure 1. As in the aligned rotator, the current sheet is a single surface that separates magnetic field lines that are attached to opposite magnetic hemispheres on the stellar surface. However, in the oblique case a pattern similar to that observed in the solar wind emerges: the sheet develops corrugations, whose amplitude increases linearly with distance from the star, as the radial wind draws out the flux lines. The current sheet now cuts the equatorial plane along twin spirals, that separate stripes of magnetic field of opposite polarity - hence the name striped wind. In the far zone, the distance between successive corrugations is small compared to the radius of curvature of their surfaces, and the spiral in the equatorial plane becomes tightly wound. Within a belt around the equator, whose thickness depends on the angle between the magnetic and rotation axes (see below), the flow locally resembles a sequence of concentric, spherical current sheets.

This picture can be made more precise by noting that, in an ideal MHD solution, the polarity of the field between two magnetic surfaces can be reversed without affecting the structure of the solution, provided a current sheet is inserted on the bounding surfaces. Bogovalov (1999) applied this argument to the split monopole solution by anchoring the inner edge of the current sheet to the obliquely rotating magnetic equator on the stellar surface. This effectively transforms the aligned split-monopole solution into one for an obliquely rotating split monopole. The resulting picture coincides precisely with that illustrated in figure 1. Recently, numerical solutions of the force-free equations have been found that have a similar appearance (Spitkovsky, 2006), although the dimensions of the calculational box extend as yet to only a few $\times r_{\mathrm{L}}$.

At high latitudes, the magnetic field does not change sign, and there are no current sheets embedded in the flow. Here, the magnetic oscillations can propagate as fast magnetosonic waves; the generation of such waves by the rotating, slightly nonaxisymmetric magnetosphere was considered by Bogovalov (2001). They could decay by nonlinear steepening leading to the formation of 
multiple shocks (Lyubarsky, 2003a). But this could release only a fraction of the Poynting flux into the plasma, since, at these latitudes, most of it is carried by the mean magnetic field.

The X-ray image of the inner Crab Nebula clearly suggests that most of the energy is transported in the equatorial belt of the pulsar wind (Aschenbach \& Brinkmann, 1975; Brinkmann et al., 1985; Weisskopf et al., 2000). The split monopole solution indeed has a pronounced maximum of the Poynting flux at the equator: $\mathrm{d} L / \mathrm{d} \Omega \propto \sin ^{2} \theta$, where $\mathrm{d} L / \mathrm{d} \Omega$ is the luminosity per solid angle interval and $\theta$ is the colatitude (Michel, 1973). In an equatorial belt, $\pi / 2-\zeta<\theta<\pi / 2+\zeta$, where $\zeta$ is the angle between the magnetic and rotation axes, the energy is carried predominantly by alternating fields (the mean field of oblique rotator vanishes in the equatorial plane). This means that most of the energy is transported in the form of a striped wind; therefore the fate of the striped wind is of special importance.

In an ideal MHD picture, the entropy wave that makes up the striped wind propagates without damping, and the dynamics is the same as in the case of the aligned rotator: in the supersonic region, the flow is essentially ballistic and propagates radially at constant speed. However, as noticed by Usov (1975), this cannot continue to arbitrarily large radius. The reason is that the amplitude of the magnetic oscillations, which is proportional to the current flowing in the sheets, decays only as $1 / R$, whereas the number of particles contained by the sheet decays as $1 / R^{2}$. At some radius, therefore, there cease to be enough particles to carry the required current.

This problem can be avoided, or at least postponed, if the current sheet is able to "recruit" additional charge carriers from the surrounding, magnetised plasma. However, such a process corresponds to the annihilation of the magnetic flux that originally threaded the newly recruited charge carriers. It hinges on the existence of an entropy creating dissipation mechanism, and the rate at which it can proceed depends on the details of the microphysics of this mechanism.

A convenient analytical approach to this problem is to employ the small wavelength approximation. This was first done by Coroniti (1990). He adopted an implicit model of the dissipation by assuming that it proceeded just fast enough to maintain the minimum required number of charge carriers. An equivalent formulation of this assumption is the requirement that the thickness of the current sheet should be equal to the gyro-radius of the sheet particles. The full set of relativistic MHD equations, complemented by this assumption about dissipation in the sheet, was solved by Lyubarsky \& Kirk (2001) in the small wavelength approximation. They found that dissipation causes the supersonic flow to accelerate, thus effectively converting Poynting flux into kinetic energy. Unfortunately, an inescapable side effect of this acceleration is the relativistic dilation of the dissipation time scale. Taking account of this, one finds that the Lorentz factor of the flow grows only slowly, according to $\Gamma \propto R^{1 / 2}$. Applying these results to the Crab Nebula, Lyubarsky \& Kirk (2001) concluded that this kind of dissipation could not convert a significant 
fraction of the wind luminosity into kinetic energy before the flow encountered the termination shock.

The implicit assumption about the dissipation rate in this calculation is clearly both fundamental and arbitrary. In an attempt to improve this situation, Kirk \& Skjæraasen (2003) compared the effects of three different prescriptions for the dissipation rate. As well as the minimum rate used by Coroniti (1990) and Lyubarsky \& Kirk (2001), they found solutions corresponding to dissipation on the timescale of the growth of the relativistic tearing mode, and to dissipation at the maximum plausible rate, governed by the transit time of sound waves across the sheet. Applying these to the Crab Nebula, they concluded that conversion of the Poynting flux to kinetic energy was indeed possible in the most favourable case, but only if the outflow carried substantially more pairs than predicted by the cascade models of Hibschman \& Arons (2001a,b).

Although the short-wavelength approximation enables one to find analytical solutions and sketch out possible scenarios for the solution of the $\sigma$ problem, it does not necessarily follow that these scenarios are realised in any given pulsar. It could be, for example, that dissipation becomes important even before the wind is accelerated to supersonic speed. The deposition of a substantial amount of energy into heat in the wind zone is likely to result in an observable signature, as discussed in section 5. Finally, if the analytical solutions indeed describe the wind accurately, it could be that relatively little Poynting flux is converted into kinetic energy before the termination shock is reached. The observed morphology of the Crab Nebula could, nevertheless, be recovered if the conversion were to take place instead in the termination shock itself (Lyubarsky, 2003b). According to the results of one-dimensional particle-in-cell simulations (Lyubarsky, 2005; Pétri \& Lyubarsky, 2007), this appears plausible, and may open up a way to understand the particle acceleration process operating at this shock front (see section 6).

\section{Observability of the wind}

\subsection{Point-like appearance}

Whereas the termination shock and nebula are visible in the X-ray (Weisskopf et al., 2000) and optical (Hester et al., 1995, 2002) images of the Crab Nebula, the wind zone they enclose appears to be dark, as was noticed in early optical images (Scargle, 1969). The standard explanation of this phenomenon is that the MHD wind is expected to be cold. In the comoving frame, a volume element in a ballistic wind would expand by a factor of $10^{18}$ between the light cylinder and the termination shock of the Crab, so that any random motion should quickly disappear. Cold, ordered motion of a highly conducting plasma, does not, however, produce synchrotron radiation. According to the ideal MHD condition, eq. (3), the acceleration of each particle vanishes, so 
that all trajectories are rectilinear. I.e., in the comoving frame, all particles are stationary. The emissivity for bremsstrahlung and for other "thermal" processes also vanishes in this case. The only possibility of producing radiation in this case is by coupling the bulk motion to the photon field. Inverse Compton scattering of photons of the cosmic microwave background or other target fields could do this, but would give rise to gamma-rays rather than X-rays or optical photons (see section 5.2). Unfortunately, the resolution of current gamma-ray detectors does not enable such photons to be distinguished from the nebular emission.

However, even if the plasma somehow remains hot, perhaps because of internal dissipation, the emission should be strongly beamed into a cone of (half)-opening angle $1 / \Gamma$ in the radial direction. One might naively expect that the image of the wind on the sky should not exceed an angular size of $1 / \Gamma$. In fact, the maximum possible size of the image is much smaller, and depends on the radial dependence of the emissivity, as well as on the radial dependence of $\Gamma$. To see this, consider a simple model in which the emissivity of the wind is such that emission from all radii $R$ is visible, provided that the radius vector $\mathbf{R}$ makes an angle of less than $1 / \Gamma(R)$ with the line of sight, and provided that $R<R_{\mathrm{T}}$, where $R_{\mathrm{T}}$ is the radius of the termination shock, here assumed, for simplicity, to be spherical in shape. The solutions found in the

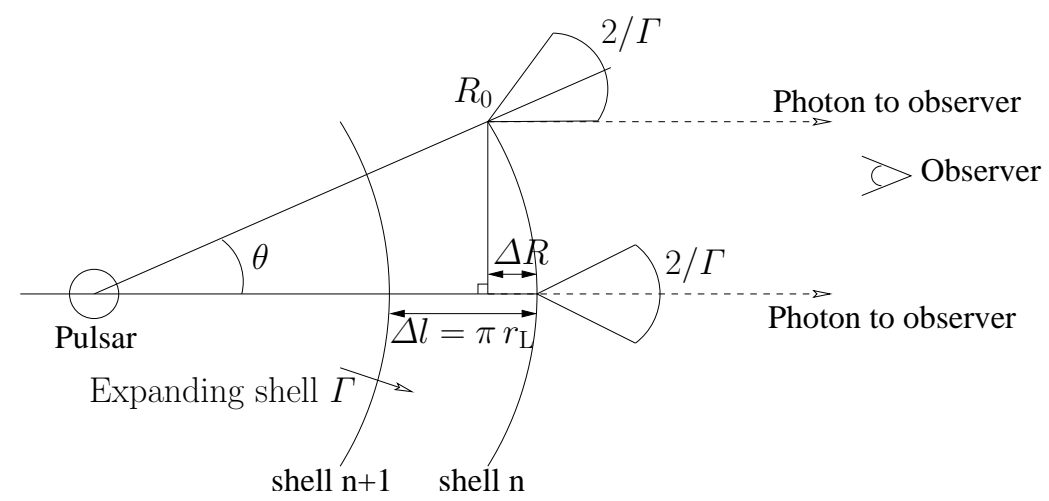

Fig. 2. Spherically symmetric shells expanding with relativistic Lorentz factor $\Gamma$ and emitting within a cone of opening angle $2 / \Gamma$ when crossing the surface $R_{0}$.

short-wavelength approximation (section 4) suggest the Lorentz factor can be parameterised as

$$
\Gamma(R)=\Gamma_{\mathrm{T}}\left(\frac{R}{R_{\mathrm{T}}}\right)^{q}
$$

where $\Gamma_{\mathrm{T}}$ is the Lorentz factor at the termination shock and $q(\geq 0)$ describes the radial acceleration. Making the reasonable assumption that the distance $D$ to the pulsar is large, specifically, $D \gg \Gamma_{\mathrm{T}} R_{\mathrm{T}}$, it follows that radiation is 
emitted in the direction of the observer provided $\sin \theta \leq 1 / \Gamma(R)$, and $R \leq R_{\mathrm{T}}$. This defines a surface that limits the visible part of the wind to:

$$
R \leq R_{\mathrm{T}} \operatorname{Min}\left[1,1 /\left(\Gamma_{\mathrm{T}} \sin \theta\right)^{1 / q}\right]
$$

On the plane of the sky, this surface appears to have an angular diameter $\alpha=\alpha_{\mathrm{T}} R \sin \theta / R_{\mathrm{T}}$ where $\alpha_{\mathrm{T}}$ is the angular diameter of the termination shock. Hence, the diameter of the visible part of the wind is

$$
\alpha \leq \frac{\alpha_{\mathrm{T}}}{\Gamma_{\mathrm{T}}} \operatorname{Min}\left[\Gamma_{\mathrm{T}} \sin \theta,\left(\Gamma_{\mathrm{T}} \sin \theta\right)^{1-1 / q}\right]
$$

For $q<1$, such as is found for all the dissipation models tested by Kirk \& Skjæraasen (2003), the maximum angular diameter of the wind emission is reached at the termination shock:

$$
\alpha_{\max }=\frac{\alpha_{\mathrm{T}}}{\Gamma_{\mathrm{T}}}
$$

whereas, for more rapidly accelerating flows $(q>1)$, the maximum diameter is determined at the launching point.

Optical observations reveal a point-like source, of angular size less than 0.1 arcsec at the position of the pulsar, whereas the termination shock has an angular diameter of approximately 12 arcsec. If there is substantial dissipation within the wind, then these observations establish a minimum Lorentz factor that is required in order that the wind should still appear point-like. For $q<1$, this is simply

$$
\begin{aligned}
\Gamma_{\mathrm{T}} & >\frac{\alpha_{\mathrm{T}}}{\alpha_{\max }} \\
& \gtrsim 100
\end{aligned}
$$

This is a modest requirement when compared to most estimates, which lie in the range from $\Gamma \sim 10^{5}$ to $10^{6}$. In the case of more rapid acceleration $q>1$, the constraint is even less stringent. For a launching point $R_{0}$ it reads $\Gamma \gtrsim 24 R_{0} / R_{\mathrm{T}} \ll 100$. Thus, one concludes that the appearance of the optical and X-ray nebula, with its central point-like source and dark region within the termination shock, does not rule out the possibility that a substantial fraction of the pulsar luminosity is dissipated and radiated away in the wind zone, provided only that the flow speed remains high. This same argument applies also to those parts of the nebula that remain in relativistic, approximately radial motion. Such regions are indeed found in two-dimensional MHD models (see section 7) and are expected to produce almost point-like images close to the pulsar.

\subsection{Inverse Compton scattering}

As mentioned above, the wind, though cold, could in principle be detected through the emission produced by inverse Compton (IC) scattering of an 
external, soft photon field (Ball \& Kirk, 2000; Bogovalov \& Aharonian, 2000). There are several possible external origins of the target photon population: the cosmic microwave background radiation, the synchrotron radiation of the nebula (dominant for the Crab from radio wavelengths to X-rays), the thermal far-infrared radiation (probably associated with dust), from the surroundings of the nebula (Atoyan \& Aharonian, 1996) and, in the case of a pulsar in a binary system, photons from the companion star (Ball \& Dodd, 2001). For a wind of constant Lorentz factor, the emission is very strongly peaked in frequency space. Even for an accelerating wind, an almost monochromatic line should appear in the gamma-ray range. The integrated (over frequency) luminosity of the scattered photons is well below the sensitivity of current gamma-ray telescopes - only if the peculiarities of the predicted spectral distribution could be exploited might it be possible to extract a signal.

Another possibility is an internal source of target photons, i.e., photons from the pulsar itself. These photons suffer the obvious disadvantage that they propagate radially outwards together with the wind. Scattering events are, therefore, likely to be almost forward in direction, which implies relatively small energy gain. However, if, by some mechanism, the wind becomes kinetic energy dominated quite close to the light cylinder, the angular momentum it must carry ensures that its velocity vector makes a substantial angle with the radius vector. Photons from the stellar surface could then be significantly boosted in energy. Bogovalov \& Aharonian (2000) investigated this possibility for the Crab, taking into account as target photons not only the thermal Xrays from the surface of this young pulsar, but also the nonthermal, pulsed emission (assuming it too comes from close to the stellar surface). Because the predicted high-energy flux depends sensitively on the location of the transition from a magnetically to a kinetically dominated wind, the observed gammaray flux (which probably originates from the nebula) puts a lower limit on this conversion radius $R_{\mathrm{w}}$. Assuming thermal X-rays as targets, they found $R_{\mathrm{w}} \geq 5 r_{\mathrm{L}}$ whereas including also the pulsed component tightens this limit to $R_{\mathrm{w}} \geq 30 r_{\mathrm{L}}$. Unfortunately, however, the constraint is not only quite weak in the sense that it does not extend far into the wind zone, but it is also sensitive to the angular dependence of the pulsar wind: if the conversion from Poynting flux to kinetic energy affects only the striped wind in the equatorial belt, the gamma-rays would be visible only to observers located in this belt, thus invalidating the constraint.

On the other hand, a lower limit on the Lorentz factor of wind follows from the fact that induced Compton scattering by the electrons in the wind does not appear to influence the radio pulses significantly. Wilson \& Rees (1978) investigated this effect in detail and estimated that, for the Crab pulsar wind, $\Gamma>10^{4}$ at a radius of roughly $100 r_{\mathrm{L}}$. 


\subsection{Pulses from the wind}

In the striped wind scenario, dissipation of magnetic energy occurs primarily in the current sheets. If this gives rise to a significant synchrotron emissivity, the resulting radiation will, as discussed above, appear to be point-like. Furthermore, provided that $R / r_{\mathrm{L}} \lesssim \Gamma^{2}$, where $R$ is the radius of the radiation source, it will be pulsed at the neutron star rotation period. This was noticed quite early in the development of pulsar theory (Michel, 1971; Arons, 1979) and has been revived recently (Kirk et al., 2002, see below). Thus, the striped wind is a possible site of production of the incoherent, high energy (optical to gamma-ray) nonthermal radiation observed from numerous pulsars. A priori, there is no compelling reason to favour this site over the inner or outer gaps in the magnetosphere. However, in contrast to these theories, the wind model has the advantage that an analytical description of the magnetic field structure is available. This removes from its predictions much of the arbitrariness present in those of the magnetospheric models.

Both the point-like appearance and the pulsations of the radiation from the wind are a consequence of relativistic beaming. Assume for simplicity that the striped wind consists of thin, concentric, spherical, radiating shells. In the equatorial plane, successive shells, $n$ and $n+1$, are separated by half a wavelength of the stripes, $\Delta l=\pi r_{\mathrm{L}}$, (see figure 2). Furthermore, assume that these shells radiate only after they cross a spherical surface of radius $R_{0}$. The maximum time delay between the arrival times at a distant observer of photons emitted on shell $n$ is $\Delta t=\Delta R / c=(1-\cos \theta) R_{0} / c$. For relativistic flows, $\theta \approx 1 / \Gamma \ll 1$. Therefore,

$$
\Delta t \approx \frac{R_{0}}{2 \Gamma^{2} c}
$$

In order to observe pulses, this time delay should be less than the time delay between emission of two consecutive shells, $n$ and $n+1$, crossing $R_{0}$, given by $\Delta T=\Delta l / c=\pi r_{\mathrm{L}} / c$. As a consequence, pulses are observed if

$$
R_{0} \lesssim 2 \pi \Gamma^{2} r_{\mathrm{L}}
$$

(cf. Arons, 1979, Eq (47)). Using the oblique split monopole solutions, Kirk et al. (2002) computed the pulse profiles expected from the striped wind. In general, two pulses per period are predicted, as observed in all gamma-ray pulsars. The spacing of these pulses is uniquely determined by the angles between the rotation axis and the magnetic axis $(\alpha)$ and the rotation axis and the line of sight $(\zeta)$. In the case of the Crab, the pulses are spaced by 0.4 of a period, consistent with $\alpha=60^{\circ}$ and $\zeta=60^{\circ}$, as independently suggested by the morphology of the X-ray torus (Ng \& Romani, 2004). The width of the main pulse and interpulse observed in the Crab is much larger than would be expected of a thin current sheet in a wind of Lorentz factor of $10^{5}$ or more. Furthermore, there is a significant unpulsed component of emission. These 
properties suggest that at least some of the radiating electrons diffuse out of the sheet.

As well as pulse profiles, the polarisation of the optical pulses from the Crab pulsar has motivated detailed comparative studies of the emission predicted by different magnetospheric models (Dyks et al., 2004; Kaspi et al., 2004). The analogous computations for the wind model have been performed by Pétri \& Kirk (2005). Extending the oblique, split-monopole solutions, these authors model the radial dependence of a current sheet of finite thickness, including an electron density that peaks in the centre of the sheet, and falls to a small, but finite value in between them, such that overall pressure balance is maintained with the varying magnetic field. An arbitrary, but small, poloidal component of the magnetic field is also added, in order to prevent the magnetic field vanishing on the neutral surface.

These extensions result in the radial dependences of magnetic field components and electron density plotted in the lower two panels of figure 3 . The thickness and relative number of electrons in each sheet is chosen to fit the observed pulse profiles, shown in the top two panels (model on the left, observations (Kanbach et al., 2003) on the right). The $B_{\theta}$ component is chosen to fit the sweep of the linear polarisation as it enters and leaves the pulse and interpulse (see the angle of polarisation $\chi$ in figure 3). The fact that $B_{\theta}$ oscillates with the same period as $B_{\varphi}$ implies that the pulsar wind itself has a small degree of circular polarisation, whose sense is determined by the sense of rotation of the neutron star, and is, therefore, the same in both the pulse and interpulse.

The detailed fits to all three observed components (intensity, degree of polarisation $\Pi$ and angle of polarisation $\chi$ ) are quite good. On the other hand, as in the magnetospheric models, a degree of arbitrariness has been introduced in order to achieve this. However, one important prediction of the wind model is independent of these uncertainties. The direction on the sky of the polarisation vector associated with the d.c. component of emission between the pulses should be determined by $B_{\varphi}$ alone, i.e., it should lie along the projection onto the sky of the rotation axis of the neutron star. This prediction is testable, because the morphology of the X-ray image of the nebula enables a symmetry axis to be identified ( $\mathrm{Ng} \&$ Romani, 2004). In figure 3 this measurement was used to orient the model predictions of the angle $\chi$. Thus, the agreement of the predicted off-pulse values of $\chi$ with the measured off-pulse polarisation direction is a strong argument in support of the wind model.

\section{The termination shock}

Pulsar wind nebulae (PWNe) are observed from the radio to $\mathrm{TeV}$ gammarays (Gaensler \& Slane, 2006). The spatially integrated spectrum contains information on the distribution in energy of the radiating particles that are presumably injected at the termination shock. The best observed example, 


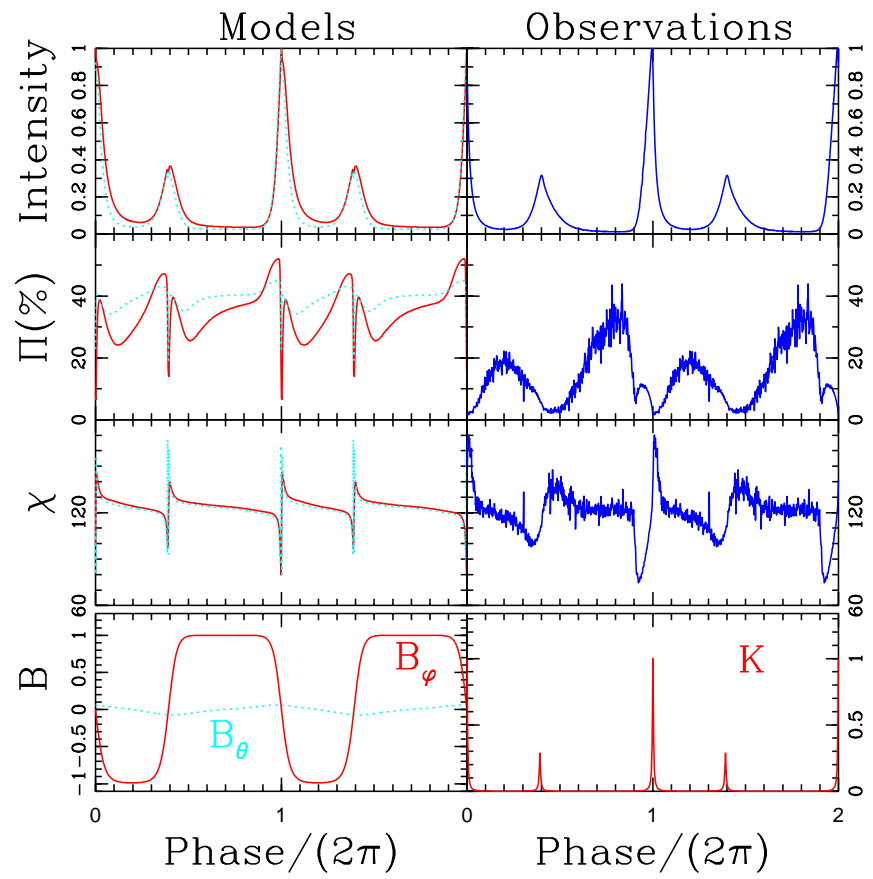

Fig. 3. Light curve of intensity, degree of polarisation and position angle of the pulsed synchrotron emission obtained for the striped wind model and measurements of these quantities for the Crab pulsar. Models with Lorentz factor $\Gamma=20$ (solid red) and 50 (dotted cyan) are shown. The bottom panels show the dependence on phase of the assumed magnetic field components $B_{\theta}$ and $B_{\varphi}$ and the particle density $K$ in the comoving frame.

the Crab Nebula, is shown in Fig. 4. Most of the radiation (from the radio up to $100 \mathrm{MeV}$ ) is synchrotron emission, and only the peak in the very-high energy gamma-ray band is attributed to the inverse Compton scattering of synchrotron photons on high-energy electrons. The synchrotron part may be described as power laws with spectral breaks around $10^{13} \mathrm{~Hz}, 10^{15} \mathrm{~Hz}$ and $100 \mathrm{keV}$. This extremely broad frequency range implies that the spectrum of relativistic electrons and positrons in the nebula extends from $\lesssim 100 \mathrm{MeV}$ to $\sim 1 \mathrm{PeV}$. The energy density (and pressure) of this relativistic plasma is dominated by leptons with an energy of around $100 \mathrm{GeV}$.

The spectra of other PWNe are generally similar to that of the Crab. In the radio band, they are hard: $\mathcal{F}_{\nu} \propto \nu^{-\alpha}$, with $\alpha$ between 0 and 0.3. At high frequencies the spectrum softens, and in the X-ray band $\alpha>1$.

Kennel \& Coroniti (1984b) postulated that the wind from the Crab pulsar has a Lorentz factor $\Gamma_{\mathrm{T}} \approx 3 \times 10^{6}$ at the termination shock, where a powerlaw particle spectrum is created at energies exceeding $E \sim m_{\mathrm{e}} c^{2} \Gamma_{\mathrm{T}} \sim 1 \mathrm{TeV}$. These electrons and positrons emit from the UV to the gamma-ray bands. Op- 


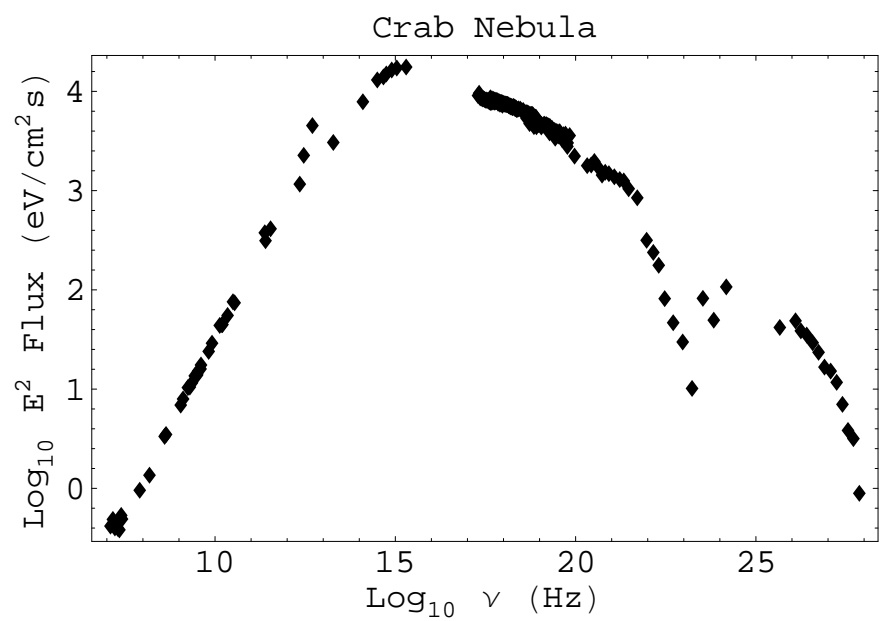

Fig. 4. The integrated spectrum of the Crab Nebula. Radio data are taken from Baars et al. (1977), infrared from Green et al. (2004), optical from Veron-Cetty \& Woltjer (1993), and X-ray through gamma-ray data (EGRET, COMPTEL and BeppoSAX) from Kuiper et al. (2001). The TeV data points $\left(>10^{25} \mathrm{~Hz}\right)$ are from H.E.S.S. observations, (Aharonian et al., 2006).

tically emitting electrons appear in the nebula as a result of the synchrotron cooling of the $\mathrm{TeV}$ electrons. The formation of the power-law spectrum at $E>m_{\mathrm{e}} c^{2} \Gamma_{\mathrm{T}}$ was considered by Hoshino et al. (1992) and Amato \& Arons (2006). They suggested that the pulsar wind is loaded by ions. Ion cyclotron waves are then collectively emitted at the shock front, and positrons and electrons are accelerated by resonant absorption of these waves. Their simulations of a relativistic shock in an electron-positron-ion plasma show that a power-law spectrum of positrons and electrons is formed. This model not only accounts for the optical to X-ray spectrum of the Crab but offers an explanation of the variable synchrotron emission of the wisps observed in the vicinity of the termination shock (Scargle, 1969; Hester et al., 2002). Gallant \& Arons (1994) and Spitkovsky \& Arons (2004) argue that the wisps arise in regions where reflection of the ions in the self-consistent magnetic field causes compressions of the electron-positron plasma and, thus, enhanced emission. The characteristic variability time-scale of the wisps (a few months) is determined in this model by the Larmor period of ions with Lorentz factors of a few $\times 10^{6}$.

The main problem with this model is that it does not account for the radio to IR emission of the nebula, which is generated by electrons and positrons with energies between $100 \mathrm{MeV}$ and $100 \mathrm{GeV}$. The large number of these electrons and positrons requires a pair injection rate of $\dot{N}=10^{40}$ to $10^{41} \mathrm{~s}^{-1}$ (Rees \& Gunn, 1974), implying $\kappa \sim 10^{6}$ (see Eq. 2). The spin-down power of the pulsar $L_{\mathrm{sd}}$, when divided by this pair output, yields a Lorenz-factor of the wind 
$\Gamma_{\mathrm{T}}=6 \times 10^{4}\left(10^{40} / \dot{N}\right)$, incompatible with the value of a few $\times 10^{6}$ required by the ion model. Kennel \& Coroniti (1984b) and Atoyan (1999) avoided this problem by assuming that the low energy electrons were injected at a very early stage of the history of the nebula. The synchrotron lifetime of the radio emitting electrons is significantly longer than the age of the nebula, so that one cannot exclude a priori that the overall spectrum depends on history of the nebula. Nevertheless, a convincing explanation of this rather convenient event in the past is lacking. Another possibility, proposed by Arons (1998), is that the radio and $\mathrm{X}$-ray emitting particles are injected by different sectors of the termination shock, X-ray particles being accelerated in the equatorial belt and the radio ones in the polar region. However, the apparent continuity of the overall spectrum of the nebula from the radio to the gamma-ray band favours for a single population of emitting electrons. Moreover, recent observations of the wisps in the radio band (Bietenholz et al., 2001, 2004) suggest that the radio emitting electrons are currently accelerated in the same region as those responsible for the optical to X-ray emission.

The spectral slope of the Crab is $\alpha=0.3$ in the radio band (Baars et al., 1977 ) and $\alpha=0.72$ in the optical (Veron-Cetty \& Woltjer, 1993), which is compatible with the assumption that the break at about $10^{13} \mathrm{~Hz}$ is due to the synchrotron burn-off effect in a magnetic field of $300 \mu \mathrm{G}$, which is close to the equipartition value (Marsden et al., 1984). The implied energy spectrum of the injected electrons and positrons has the form $N(E) \propto E^{-1.6}$. This view is supported by Gallant \& Tuffs $(2000,2002)$ who found that the infra-red spectral index in the central parts of the Crab is close to that in the radio, and gradually steepens as one moves outwards. The high frequency break lies in the ultra-violet band at about a few $\times 10^{15} \mathrm{~Hz}$, which implies that at $E>E_{\mathrm{br}} \sim 1 \mathrm{TeV}$ the injection spectrum becomes steeper ${ }^{5}$; the spectral slope in the X-ray band, $\alpha=1.1$, corresponds, when the synchrotron burn-off effect is taken into account, to an injection spectrum of $N(E) \propto E^{-2.2}$ at $E>E_{\mathrm{br}}$, so that the injection spectrum is a broken power-law. Taking into account that no sign of a low frequency cut-off is observed in the Crab spectrum down to about $30 \mathrm{MHz}$ (at lower frequencies the spectrum is dominated by the pulsar), one concludes that the injection spectrum of electrons and positrons extends down to $E_{\min } \leq 100 \mathrm{MeV}$.

Such an injection spectrum implies a rather unusual acceleration process. The energy density of the injected plasma, $\int E N(E) \mathrm{d} E$, is dominated by particles with $E \sim E_{\mathrm{br}}$ whereas most of the particles find themselves at $E \sim E_{\min } \ll E_{\mathrm{br}}$ so that the acceleration process should somehow transfer most of the total energy of the system to a handful of particles leaving for the majority only a small fraction of the energy. This is not what one would

\footnotetext{
${ }^{5}$ Del Zanna et al. (2006) argue that the UV break is due to the synchrotron cooling, which assumes that the magnetic field in the nebula remains a few times less than the equipartition level. In this case, the $10^{13} \mathrm{~Hz}$ break should be attributed to the break in the injection spectrum so that $E_{\mathrm{br}} \sim 100 \mathrm{GeV}$.
} 
normally expect from a shock-associated acceleration process, where the particle flow is randomised at the shock and only a fraction of the upstream kinetic energy is converted into the energy of a few accelerated particles. In this case, the temperature $T$ downstream roughly corresponds to the upstream particle kinetic energy and the high-energy tail of accelerated particles merges, at its low energy end, with the quasi-thermal distribution at $E \sim$ few $\times k_{\mathrm{B}} T$. For the Crab, this means that the Lorentz factor of the wind at the termination shock, $\Gamma_{\mathrm{T}}$, should not exceed a few hundred in order to deliver the majority of the electrons and positrons into the downstream region with $E_{\min } \sim 100$ $\mathrm{MeV}$. On the other hand, the particle energy spectrum implies that the energy per electron in the system is much larger, about $10 \mathrm{GeV}$, so there would appear to exist an energy reservoir in the flow that eventually dissipates in a small fraction of the particles.

Gallant et al. (2002), modifying the original idea of Hoshino et al. (1992), suggested that the wind is loaded by so many ions that their kinetic energy dominates the wind energy flux. At the shock front, the pairs are thermalized with $k_{\mathrm{B}} T \sim m_{\mathrm{e}} c^{2} \Gamma_{\mathrm{T}} \leq 100 \mathrm{MeV}$ whereas the ions collectively emit about one half of their energy as cyclotron waves. The radio emitting electrons and positrons are accelerated by these waves according to the mechanism by Hoshino et al. (1992) and Amato \& Arons (2006). The problem with this theory, is that the required injection rate of ions, $\sim L_{\mathrm{sd}} /\left(m_{\mathrm{p}} c^{2} \Gamma_{\mathrm{T}}\right) \sim 10^{39} \mathrm{~s}^{-1}$, vastly exceeds the fiducial Goldreich-Julian elementary charge loss rate, $\dot{N}_{\text {GJ }} \sim 10^{34} \mathrm{~s}^{-1}$, obtained by substituting $\kappa=1$ into eq. (2). It is difficult to imagine how the electric field at the surface of the star could extract ions with a density orders of magnitude higher than that required to screen out this same field. On the other hand, one cannot exclude by observation that pulsars emit the required number of ions. Their presence in the plasma injected into the nebula could, in principle, be revealed by observations of ultra high energy gamma-rays and/or neutrinos (Amato et al., 2003; Bednarek, 2003; Bednarek \& Bartosik, 2003); the data currently available on the Crab Nebula are, however, compatible with the assumption that all the observed emission is generated by electrons and positrons and no relativistic ions are present (Aharonian et al., 2006). However, it has recently been proposed that the high energy emission of the PWN Vela X can be understood if a significant fraction of the pulsar spin-down power is carried by relativistic nuclei (Horns et al., 2006).

Another possible energy reservoir for particle acceleration is present if most of the pulsar spin-down energy is still stored in the striped magnetic field when the flow enters the termination shock (Lyubarsky, 2003b; Pétri \& Lyubarsky, 2007). As discussed in section 4, this would be expected if the microphysics of the dissipation process proceeds relatively slowly. The flow then accelerates only slowly, and the Lorentz factor at the termination shock is compatible with a low $E_{\min }$ in the energy distribution of the accelerated particles. When the flow enters the shock, the alternating magnetic fields annihilate transferring the energy to the particles and one can speculate that 
the radio-to-optical emission of $\mathrm{PWNe}$ is generated by electrons and positrons accelerated in the course of reconnection of the alternating magnetic field at the pulsar wind termination shock. One-dimensional PIC simulations indeed show that the alternating fields easily annihilate at the shock (Lyubarsky, 2005; Pétri \& Lyubarsky, 2007), but do not show evidence of a nonthermal distribution. This may be attributed to a highly idealised one-dimensional field structure in the simulations. In the real case, reconnection is expected to occur at X-points, and particles gain energy according to how close they approach such a point. Higher dimensional studies of the reconnection process in a relativistic electron-positron plasma without a striped field (Romanova \& Lovelace, 1992; Zenitani \& Hoshino, 2001, 2005a,b; Larrabee et al., 2003) demonstrate efficient acceleration of non-thermal particles. However, even though the obtained spectra can be roughly described by a power-law with an exponential cutoff, there is as yet no evidence of a hard power-law spectrum over the wide energy range observed in PWNe.

The formation of the high-energy tail in the spectrum at $E>E_{\mathrm{br}}$, on the other hand, can be attributed to the first-order Fermi acceleration mechanism. In the absence of strong cross-field diffusion, this mechanism does not operate at a perpendicular shock (where the magnetic field is perpendicular to the shock normal), because particles are prevented from diffusing back upstream by the fact that their guiding centres must follow the field lines, which run parallel to the shock front. Because the perpendicular field component is compressed in the proper frame by a large factor, relativistic shocks almost always fall into this category (Begelman \& Kirk, 1990). However, in reality, some degree of cross field transport must occur, and the question of whether or not the first-order mechanism operates at a relativistic shock hinges on the relative magnitudes of the ordered magnetic field and the stochastic component that produces the cross-field transport. Simulations of acceleration in prescribed stochastic fields indeed show that acceleration is less effective for stronger ordered fields (Ostrowski \& Bednarz, 2002), and corroborating evidence is beginning to accumulate from $3 \mathrm{D}$, relativistic, particle-in-cell simulations (Spitkovsky, 2005). On the other hand, in the pulsar case, annihilation of the ordered magnetic field in the wind (or in the shock) may leave a highly turbulent, small-scale magnetic field, whose chaotic component significantly exceeds the regular field (Reville et al., 2006). Particle scattering off these strong inhomogeneities may then allow the first-order Fermi mechanism to operate. It is interesting that the first-order Fermi mechanism operating at an ultra-relativistic shock yields, in the case of isotropic diffusion of the accelerated particles, an energy distribution $E^{-2.2}$ (Bednarz \& Ostrowski, 1998; Kirk et al., 2000; Achterberg et al., 2001) - exactly the value required to explain the X-ray spectrum of the Crab. 


\section{The nebula}

The physical conditions in pulsar winds, though difficult to determine directly, can be inferred from the observed properties of PWNe, which are simply the bubbles inflated by the wind in the surrounding medium. It is the wind plasma that fills the nebula and produces the observed nonthermal electromagnetic emission. The typical size of the PWN is a few parsecs. Specifically, the Crab Nebula is an ellipsoid of projected dimension $2 \mathrm{pc} \times 3 \mathrm{pc}$.

The nebula is confined by its nonrelativistic surroundings, and the termination shock of the pulsar wind is located at a radius defined by the condition that the confining pressure balances the momentum flux of the wind. In the case of the Crab Nebula, the shock radius was estimated by Rees \& Gunn (1974) to be $0.1 \mathrm{pc}$, in excellent agreement with the radius of the apparent central hole in the nebula brightness distribution (Scargle, 1969). At the shock front, the wind energy is released into the relativistic particles responsible for the observed radiation. Rapidly moving wisps and variable knots were found in this region (Scargle, 1969; Hester et al., 1995, 2002), confirming it as the site of energy injection into the nebula.

Early spherically symmetrical MHD models of the Crab Nebula (Rees \& Gunn, 1974; Kennel \& Coroniti, 1984a,b; Emmering \& Chevalier, 1987) seem to describe its main properties perfectly well. The observed brightness and the spectral index distributions are generally consistent with the assumption that the relativistic particles are injected by the pulsar in the centre of the nebula and then expend their energy on synchrotron emission and $p \mathrm{~d} V$ work (Kennel \& Coroniti, 1984b; Amato et al., 2000). The size of the nebula decreases with observed frequency because the higher the energy of a particle, the faster it loses energy by synchrotron emission. The synchrotron life-time of the radio emitting electrons is larger than the age of the nebula. Therefore, they fill the whole nebula. The life-time of the optically emitting electrons is comparable or somewhat shorter than the age of the nebula and, therefore, the optical image is smaller than the radio one. The X-ray emitting electrons lose their energy in only a few years. Therefore, X-rays are emitted only close to the pulsar. This makes the X-rays an exceptional tool for the study of the interaction of the pulsar wind with the nebula. Whereas the radio and optical images are dominated by the outer parts of the nebula and distorted by interaction with the surroundings, the X-rays trace the freshly injected plasma and reveal the physical processes in the very heart of the nebula.

The basic conclusion of these early models was that the pulsar wind must be particle dominated. The magnetic field in the lab. frame increases by a factor of roughly three across a relativistic shock front. In the subsonic postshock flow, the plasma density remains approximately constant, so that conservation of the magnetic flux within a radially expanding magnetic loop implies linear growth of the field strength with radius. In order for the magnetic pressure at the outer boundary of the nebula not to exceed the plasma pressure, the magnetisation of the wind just upstream of the termination shock should be 
at most $\sigma \sim 3 \times 10^{-3}$ (Kennel \& Coroniti, 1984a; Emmering \& Chevalier, 1987). Such a low value of $\sigma$ is puzzling, because the pulsar wind is launched as a Poynting dominated flow with $\sigma \gg 1$, and, as discussed in sections 3 and 4 , it is not easy to invent a realistic mechanism to reduce $\sigma$ to the required level.

On the other hand, $\sigma$ cannot be significantly lower than the above value, because magnetic stresses play an important role in shaping the nebula: the observed elongation is convincingly explained as the result of pinching by an azimuthal magnetic field (Begelman \& Li, 1992; van der Swaluw, 2003). The discovery of the jet-torus structure confirmed that the nebula is elongated along the pulsar spin axis so that the magnetic field in the nebula should indeed be wrapped around the major axis of the ellipsoid.

The jet-torus structure was revealed already in earlier X-ray observations (Aschenbach \& Brinkmann, 1975; Brinkmann et al., 1985; Hester et al., 1995) and was clearly resolved by Chandra (Weisskopf et al., 2000; Hester et al., 2002; Mori et al., 2004). Similar structures have been found in other PWNe (for a review, see Gaensler \& Slane, 2006), suggesting that this is a generic phenomenon. The inner boundary of the X-ray torus of the Crab coincides with the position of the standing shock predicted by early spherically symmetric models, but the morphology of the inner nebula forces one to abandon the assumption of spherical symmetry. The observed structure suggests that the pulsar wind itself is highly anisotropic, with most of the energy being transported in the equatorial belt. Encouragingly, simple (split-monopole) models of the pulsar wind indeed have the property that the energy flux reaches a maximum at the equator (see section 4). However, the origin of the polar jet is not so evident.

The jet in the Crab Nebula, as well as the jets in other PWNe, appears to originate from the pulsar and propagate along the rotation axis. This seems to indicate that they are a part of the pulsar wind, possibly collimated by magnetic hoop stresses. However, as discussed in section 3, this mechanism does not work in ultra-relativistic flows. Moreover, such a jet would presumably be ultrarelativistic, whereas the observed jets certainly are not, as follows both from direct measurements of the proper motion in the jets of the Crab and Vela (Hester et al., 2002; Pavlov et al., 2003), and from the fact that both the jet and counter-jet are visible. Lyubarsky (2002) pointed out that magnetic collimation, though ineffective in the pulsar wind, could be responsible for the formation of the jets beyond the termination shock. In the equatorial belt, which carries most of the energy, annihilation of the striped field (see section 4) ensures that the residual magnetic field is low and does not affect the dynamics of the post-shock plasma. However, the magnetisation of the high latitude flow remains significant. This naturally results in the separation of the post-shock flow into an equatorial disk and a magnetically collimated polar outflow. In this model, the observed jet arises as a result of the axial compression of the shocked plasma. 
In the split monopole model of the pulsar wind, the angular distribution of the total energy flux $f_{\text {tot }}$ can be written as

$$
f_{\mathrm{tot}}=\frac{f_{0}}{R^{2}}\left(\sin ^{2} \theta+1 / \sigma_{0}\right)
$$

(Michel, 1973; Bogovalov, 1999), where $R$ and $\theta$ are the usual spherical coordinates, $f_{0}$ and $\sigma_{0} \gg 1$ constants. The first term in parentheses represents the Poynting flux, whereas the second one accounts for the small initial contribution of particles. As discussed above, a significant part of the Poynting flux is transferred by variable fields, and can be converted into plasma energy relatively easily. But the total energy per particle is conserved along the streamlines and, as they are radial, the angular distribution (18) remains unchanged. Lyubarsky (2002) showed that in such a strongly anisotropic wind, the termination shock is highly aspherical, forming a cusp on the axis of the flow. Therefore, the jet could appear to originate from the pulsar simply because the termination shock lies much closer to the pulsar in the polar regions than in the equatorial belt. By neglecting magnetic stresses in the vicinity of the shock, Bogovalov \& Khangoulian (2002) independently found that the subsonic region lies much closer to the pulsar in the polar region than in the equatorial plane. However, the formation of a jet on the axis depends on the additional effect of magnetic collimation (Khangoulian \& Bogovalov, 2003).

To test this qualitative picture Komissarov \& Lyubarsky $(2003,2004)$ performed axisymmetric relativistic MHD simulations of a flow produced by an anisotropic pulsar wind within a slowly expanding cavity of cold heavy gas. They adopted eq. (18) for the angular distribution of the total energy in the wind and assumed that all waves decayed either in the wind or at the termination shock so that the postshock flow is completely determined by the total energy flux, $f_{\text {tot }}$ and the mean field, $B$, in the wind. As the postshock MHD parameters are independent of where exactly the waves decay, one can assume for simplicity that all the wave energy has already been converted into the flow kinetic energy on entering the shock, $\rho \Gamma v=f_{\text {tot }}-c B^{2} / 4 \pi$. For an ultra-relativistic flow, $\Gamma \gg 1$, the post-shock plasma is relativistically hot and the dynamics of the downstream flow depends only on the total energy flux and magnetisation, i.e., it does not depend on $\rho$ and $\Gamma$ separately, but only on their product. Therefore, the flow is determined by the two functions, $f_{\mathrm{tot}}(\theta)$ and $B(\theta)$.

The distribution of the mean field in the pulsar wind is not known but certainly goes to zero on the equator of a flow driven by an obliquely rotating, centred dipole. Moreover the mean field vanishes on the axis of the flow, because an unphysical singular current would otherwise be required. Taking into account that the field is frozen into a radial flow and, therefore, the radial dependence is given by eq. (10), Komissarov \& Lyubarsky $(2003,2004)$ chose a simple model for the mean field:

$$
B=\sqrt{\frac{4 \pi f_{0}}{c}} \frac{\xi}{R} \sin \theta\left(1-\frac{2 \theta}{\pi}\right)
$$


where the free parameter $\xi \leq 1$ controls the magnetisation of the wind. The ratio of the energy transported by the mean electromagnetic field to the energy transported by the particles is $\sigma=0.1 \xi^{2}$.

The results of these simulations are shown in Fig. 5. The termination shock is highly aspherical being significantly closer to the pulsar in the polar zone than in the equatorial plane. Most of the downstream flow is initially confined to the equatorial plane. The magnetic hoop stresses stop the outflow in the surface layers of this equatorial disk and redirect it into magnetically confined polar jets, which, therefore, are formed outside of the termination shock. Velocities both in the disk and in the jet were found to be about $0.5 c$, close to those inferred from observation (Hester et al., 2002; Pavlov et al., 2003; DeLaney et al., 2006).

Images of the nebula in synchrotron emission were also simulated, taking into account the relativistic beaming effect and particle energy losses (Fig. 6). These images resemble those of the Crab and other PWNe obtained by Chandra. They exhibit both a system of rings, giving the impression of an equatorial disk-like or even toroidal structure, and well-collimated polar jets, which give the illusion of originating directly from the pulsar.

The simulated images also reveal a bright central source. At high latitudes, plasma enters the shock highly obliquely, and, therefore, the post-shock velocity remains high, $\sim 0.9 c$ and is not deflected far from its upstream radial direction. As discussed in section 5.1, this results in an almost point-like feature close to the pulsar. However, it is not connected with emission from the Crab pulsar itself, but may be identified with the bright knot discovered by Hester et al. (1995) and located 0.65 arcsec to the southeast.

Del Zanna et al. (2004) investigated the sensitivity of the morphology of the nebula to the angular dependence of the wind magnetisation. They simulated the evolution of the pulsar wind nebula within the expanding supernova ejecta adopting Eq. (18) for the total energy flux, but parameterising the angular dependence of the mean magnetic field as $B \propto \sin \theta \tanh [b(\pi / 2-\theta)]$ (cf. eq. (19)). The effect on the flow of the width of the low magnetisation region in the equatorial belt (associated with the striped wind) was investigated by varying $b$. They found that collimation occurs at any $b$ but that the overall picture of the flow changes completely if there is no belt of low magnetisation, i.e. when $b \rightarrow \infty$.

In this case, the magnetic hoop stresses in the equatorial plane completely suppress the radial flow after a few termination shock radii, diverting the plasma towards the axis. A part of the flow then enters a polar outflow whereas another part is directed towards the pulsar forming a large-scale vortex. The polar outflow is quite wide (the radius is comparable with the equatorial radius of the termination shock) and is formed rather far from the pulsar. The outflow starts at a distance larger than the equatorial radius of the termination shock, whereas, below the base of the outflow, the plasma moves towards the pulsar. A wide outflow starting far from the pulsar was also found by Bogovalov et al. (2005) who simulated the nebula formed by a pulsar wind with $B \propto \sin \theta$. This 


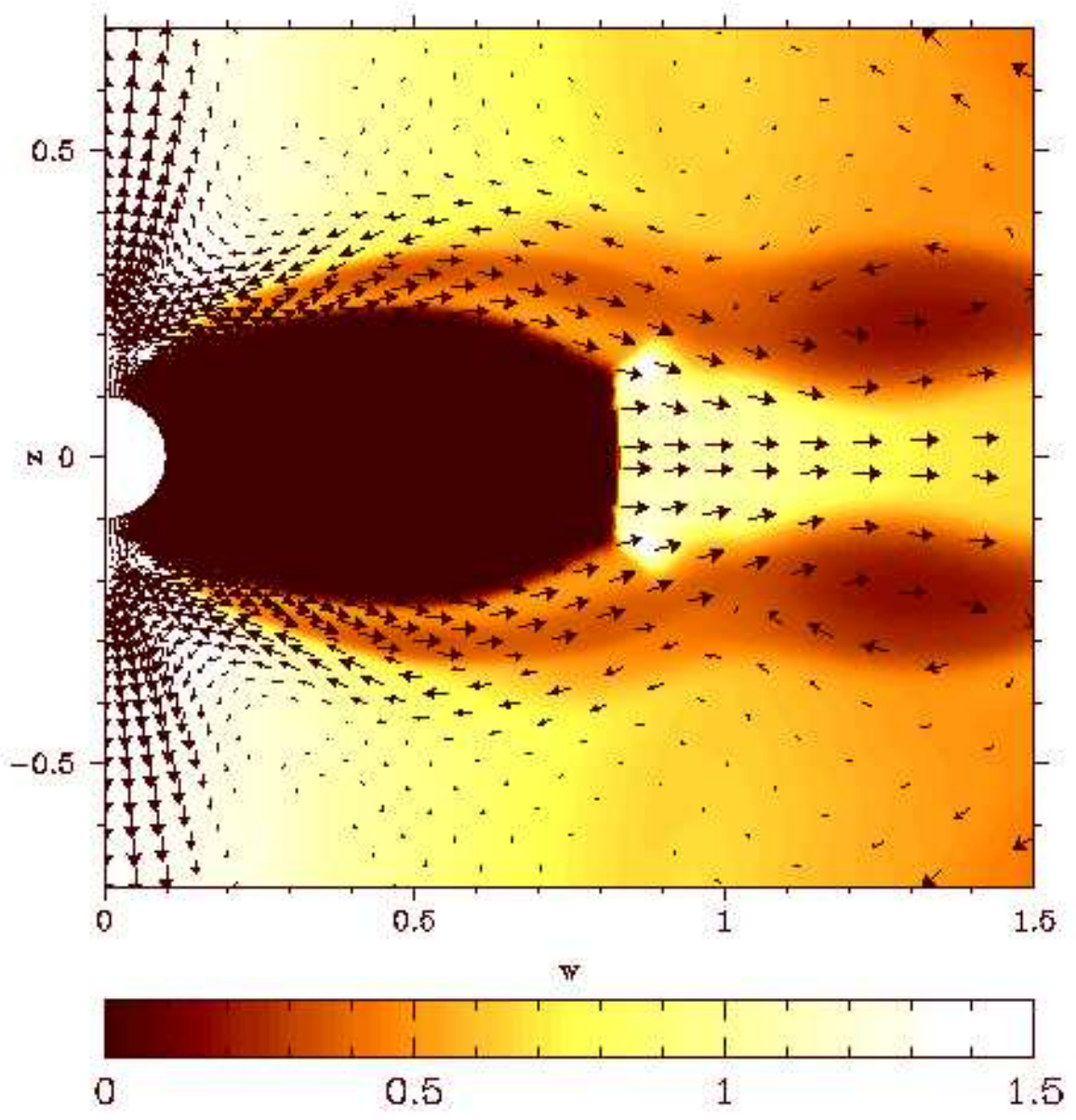

Fig. 5. The flow around the termination shock (Komissarov \& Lyubarsky, 2003). The flow velocity is shown by arrows, the plasma energy density by colour.

picture does not match the observed structure of the nebula, where the jet is very narrow and appears to start close to the pulsar.

The clearly observed polar-equator dichotomy arises only if the magnetisation is low in the equatorial belt of the pulsar wind; according to Del Zanna et al. (2004), $b=10$ is enough. Only in this case is the equatorial outflow not significantly affected by the magnetic stresses, and is able to extend deep into the nebula, whilst the high latitude wind is collimated into a narrow polar jet. In this sense, the jet-torus structure provides evidence in favour of magnetic dissipation in the striped wind. One can speculate that the smaller the angle between the magnetic and rotation axes of the pulsar, the narrower 


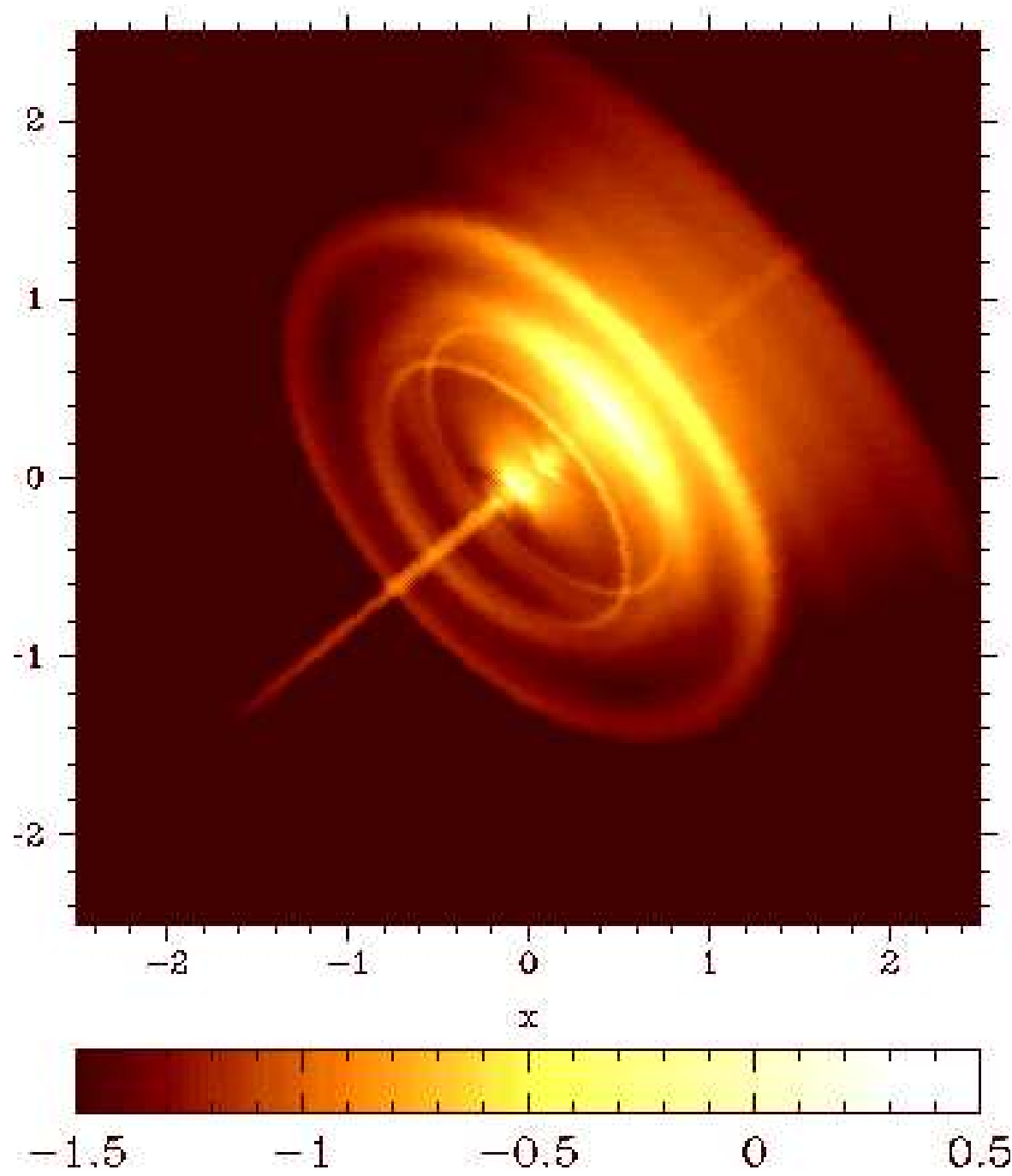

Fig. 6. The simulated image of the synchrotron emission of the Crab Nebula (Komissarov \& Lyubarsky, 2003).

the striped zone and, therefore, the larger is the fraction of the flow that is collimated into a polar jet.

The MHD model thus captures many properties of the Crab Nebula quite well and, in spite of some quantitative differences with the observational data, is probably basically correct. By incorporating more physics into the model, one can hope to achieve even better agreement with the observations and infer the parameters of the pulsar wind in different systems. Thus, Bucciantini 
et al. (2005b) computed polarisation maps of the nebula, and Del Zanna et al. (2006) investigated the effects of synchrotron cooling on the images and spectral properties of PWNe. Bucciantini \& Del Zanna (2006) addressed the development of the Kelvin-Helmholtz instability in the disk outflow and the resulting small-scale modulation of the synchrotron radiation. Global variations of the flow are also clearly visible in simulations; Bogovalov et al. (2005) has reported quasiperiodic oscillations on timescales from years to dozens of years, but the origin of these variations is still unclear.

The interaction of a PWN with the surrounding gas is another phenomenon that can be studied using relativistic MHD simulations, and the results are a valuable tool in the interpretation of the wide variety shown by X-ray images. Thus, the evolution of the nebula within the expanding supernova ejecta, including interaction with the reverse shock, was simulated by van der Swaluw et al. (2001) in a spherically symmetric model, and Bucciantini et al. (2004) studied the Rayleigh-Taylor instability at the interface between an expanding PWN and its surrounding supernova remnant. Bucciantini et al. (2005a) presented axisymmetric simulations of pulsar wind bow-shock nebulae arising around pulsars, that have already emerged from the progenitor supernova remnant.

These detailed results are strongly dependent on the angular structure of the pulsar wind. Recent progress in simulating the obliquely rotating dipole magnetosphere and wind (Spitkovsky, 2006), suggests that a more realistic model of the angular dependence of the pulsar wind might soon be available to replace parameterisations such as eqs. (18) and (19). Then one might even hope to constrain the angle between the rotation and magnetic axes of the pulsar from the observed structure of the inner nebula.

All PWNe models discussed so far are either spherically or axially symmetric. However, in MHD flows with non-negligible magnetic stresses, 3D effects could come into play because the underlying symmetric configurations could be unstable. Inadequacy of the axisymmetric picture may be suggested by the fact that all models of the Crab Nebula require a very low overall magnetisation $\sigma \approx 0.01$ - if the magnetisation were larger the nebula would be distorted by the pinch effect beyond observational limits. However, the ideas on magnetic dissipation discussed in section 4 are capable of removing only the oscillating part of the magnetic field in the equatorial belt. This apparent problem can be alleviated if, as suggested by Begelman (1998), the kink instability destroys the concentric field structure in the nebula. In the axisymmetric case, magnetic loops in the expanding flow are forced to expand and perform work against the magnetic tension. The kink instability allows the loops to come apart and one can expect that in $3 \mathrm{D}$, the hoop stress would be less effective than suggested by axisymmetric simulations. These stresses cannot disappear altogether, because they are responsible for driving the kink instability itself. Nevertheless, this effect could have important implications for the inferred magnetisation of the pulsar wind. 


\section{Summary}

The suggestions of Piddington (1957) that the relativistic particles and mag-

netic fields in PWNe originate in a central stellar object, as well as that of Pacini (1967) that the ultimate energy source lies in the rotational energy of the neutron star, have both stood the test of time and the scrutiny of increasingly high resolution observations at all accessible wavelengths. We also now know that the pulsar wind has differing equatorial and polar components, as suggested by Aschenbach \& Brinkmann (1975). The Crab Nebula, in particular, can be quite well described by an axisymmetric, relativistic MHD model. This establishes without much doubt that the energy is injected into the nebula at the wind termination shock, with most of it being concentrated into an equatorial belt in a particle dominated form, and that the jets consist of shocked plasma collimated by azimuthal fields, rather than a polar wind.

Consensus is more difficult to achieve in the case of the wind. That it is highly relativistic on entering the termination shock, with bulk Lorentz factor $\Gamma_{\mathrm{T}}>100$, seems secure. This lower limit, which applies only if the flow remains magnetically dominated up to the shock front, is still controversial; other models generally place $\Gamma_{\mathrm{T}}$ between $10^{4}$ and $10^{6}$. That the equatorial flow is born as a "striped wind" (Michel, 1971; Coroniti, 1990) seems likely. That it is almost perfectly radial and accelerates at least to the fast magnetosonic point is also not controversial.

Perhaps the most puzzling open question is the matter content of the wind. Detailed models of pair creation in the magnetosphere produce far too few leptons to explain all of the nebular emission. The ion content is also puzzling: observations of the wisps or, alternatively, of the integrated spectrum, could be nicely explained if ions contributed to the energy flux, but they are even harder to extract from the pulsar.

The much discussed $\sigma$-problem also remains an open question, especially for the Crab Nebula. Although two solutions work for the equatorial part of the wind (dissipation of the stripes either in the wind or at the termination shock), neither of these works in the polar wind region. The only possibilities here seem to be either that the equatorial belt is initially very broad (i.e., a highly oblique rotator) or that kink instabilities in the outer nebula ultimately release the magnetic tension.

The particles responsible for the nonthermal emission are almost certainly accelerated at the termination shock front. However, which mechanisms are responsible is controversial. It seems that at least two different ones must be operating, one at low energies, (100 MeV to $1 \mathrm{TeV}$ ) which may be either resonant absorption of coherently emitted ion cyclotron waves, or the annihilation of magnetic flux in the shock front, and one at high energy $(>1 \mathrm{TeV})$ which is most likely the first order Fermi mechanism. However, none of these mechanisms can be regarded as fully worked out from a theoretical point of view. 
Progress, as always, will flow from more and improved observations. For the nebula, X-ray and optical images continue to be of crucial importance, and maps in the $\mathrm{TeV}$ band are just starting to appear (Aharonian et al., 2005). For the wind, the observational signatures will be point-like, and might be pulsed. High time-resolution optical polarimetry of pulsar emission will have important input on the question of the location of the pulse-forming region. Finally, the large number of new gamma-ray pulsars that will be discovered by GLAST is also highly likely to lead to the extinction of some theories of high energy pulsed emission, as well as providing much more accurate spectra for comparison with models of PWNe.

Acknowledgement. This work was supported by a grant from the G.I.F. the GermanIsraeli Foundation for Scientific Research and Development

\section{References}

Achterberg, A., Gallant, Y. A., Kirk, J. G., \& Guthmann, A. W. 2001, MNRAS, 328, 393

Aharonian, F., Akhperjanian, A. G., Aye, K.-M., et al. 2005, A\&A, 435, L17 Aharonian, F., Akhperjanian, A. G., Bazer-Bachi, A. R., et al. 2006, A\&A, 457,899

Amato, E. \& Arons, J. 2006, ApJ, 653, 325

Amato, E., Guetta, D., \& Blasi, P. 2003, A\&A, 402, 827

Amato, E., Salvati, M., Bandiera, R., Pacini, F., \& Woltjer, L. 2000, A\&A, 359,1107

Arons, J. 1979, Space Science Reviews, 24, 437

Arons, J. 1998, Memorie della Societa Astronomica Italiana, 69, 989

Aschenbach, B. \& Brinkmann, W. 1975, A\&A, 41, 147

Asseo, E., Kennel, C. F., \& Pellat, R. 1978, A\&A, 65, 401

Atoyan, A. M. 1999, A\&A, 346, L49

Atoyan, A. M. \& Aharonian, F. A. 1996, MNRAS, 278, 525

Baars, J. W. M., Genzel, R., Pauliny-Toth, I. I. K., \& Witzel, A. 1977, A\&A, 61,99

Ball, L. \& Dodd, J. 2001, Publications of the Astronomical Society of Australia, 18, 98

Ball, L. \& Kirk, J. G. 2000, Astroparticle Physics, 12, 335

Bednarek, W. 2003, A\&A, 407, 1

Bednarek, W. \& Bartosik, M. 2003, A\&A, 405, 689

Bednarz, J. \& Ostrowski, M. 1998, Physical Review Letters, 80, 3911

Begelman, M. C. 1998, ApJ, 493, 291

Begelman, M. C. \& Kirk, J. G. 1990, ApJ, 353, 66

Begelman, M. C. \& Li, Z.-Y. 1992, ApJ, 397, 187

Beskin, V. S., Kuznetsova, I. V., \& Rafikov, R. R. 1998, MNRAS, 299, 341 
Beskin, V. S. \& Nokhrina, E. E. 2006, MNRAS, 367, 375

Bietenholz, M. F., Frail, D. A., \& Hester, J. J. 2001, ApJ, 560, 254

Bietenholz, M. F., Hester, J. J., Frail, D. A., \& Bartel, N. 2004, ApJ, 615, 794

Bogovalov, S. \& Tsinganos, K. 1999, MNRAS, 305, 211

Bogovalov, S. V. 1999, A\&A, 349, 1017

Bogovalov, S. V. 2001, A\&A, 367, 159

Bogovalov, S. V. \& Aharonian, F. A. 2000, MNRAS, 313, 504

Bogovalov, S. V., Chechetkin, V. M., Koldoba, A. V., \& Ustyugova, G. V. 2005, MNRAS, 358, 705

Bogovalov, S. V. \& Khangoulian, D. V. 2002, MNRAS, 336, L53

Brinkmann, W., Aschenbach, B., \& Langmeier, A. 1985, Nature, 313, 662

Bucciantini, N., Amato, E., Bandiera, R., Blondin, J. M., \& Del Zanna, L. 2004, A\&A, 423, 253

Bucciantini, N., Amato, E., \& Del Zanna, L. 2005a, A\&A, 434, 189

Bucciantini, N. \& Del Zanna, L. 2006, A\&A, 454, 393

Bucciantini, N., del Zanna, L., Amato, E., \& Volpi, D. 2005b, A\&A, 443, 519

Buckley, R. 1977, MNRAS, 180, 125

Chiueh, T., Li, Z.-Y., \& Begelman, M. C. 1998, ApJ, 505, 835

Contopoulos, I. \& Kazanas, D. 2002, ApJ, 566, 336

Contopoulos, I., Kazanas, D., \& Fendt, C. 1999, ApJ, 511, 351

Coroniti, F. V. 1990, ApJ, 349, 538

Davis, L. 1947, Physical Review, 72, 632

Del Zanna, L., Amato, E., \& Bucciantini, N. 2004, A\&A, 421, 1063

Del Zanna, L., Volpi, D., Amato, E., \& Bucciantini, N. 2006, A\&A, 453, 621

DeLaney, T., Gaensler, B. M., Arons, J., \& Pivovaroff, M. J. 2006, ApJ, 640, 929

Dyks, J., Harding, A. K., \& Rudak, B. 2004, ApJ, 606, 1125

Emmering, R. T. \& Chevalier, R. A. 1987, ApJ, 321, 334

Gaensler, B. M. \& Slane, P. O. 2006, Ann. Rev. Astron. Astrophys., 44, 17

Gallant, Y. A. \& Arons, J. 1994, ApJ, 435, 230

Gallant, Y. A. \& Tuffs, R. J. 2000, in ASP Conf. Ser. 202: IAU Colloq. 177: Pulsar Astronomy - 2000 and Beyond, ed. M. Kramer, N. Wex, \& R. Wielebinski, 503-+

Gallant, Y. A. \& Tuffs, R. J. 2002, in ASP Conf. Ser. 271: Neutron Stars in Supernova Remnants, ed. P. O. Slane \& B. M. Gaensler, 161-+

Gallant, Y. A., van der Swaluw, E., Kirk, J. G., \& Achterberg, A. 2002, in ASP Conf. Ser. 271: Neutron Stars in Supernova Remnants, 99-104

Green, D. A., Tuffs, R. J., \& Popescu, C. C. 2004, MNRAS, 355, 1315

Gruzinov, A. 2005, Physical Review Letters, 94, 021101

Harding, A. K. 2005, in AIP Conf. Proc. 801: Astrophysical Sources of High Energy Particles and Radiation, ed. T. Bulik, B. Rudak, \& G. Madejski, 241-252

Harding, A. K. \& Lai, D. 2006, Reports of Progress in Physics, 69, 2631

Hester, J. J., Mori, K., Burrows, D., et al. 2002, ApJ, 577, L49

Hester, J. J., Scowen, P. A., Sankrit, R., et al. 1995, ApJ, 448, 240 
Hibschman, J. A. \& Arons, J. 2001a, ApJ, 554, 624

Hibschman, J. A. \& Arons, J. 2001b, ApJ, 560, 871

Hones, Jr., E. W. \& Bergeson, J. E. 1965, JGR, 70, 4951

Horns, D., Aharonian, F., Santangelo, A., Hoffmann, A. I. D., \& Masterson, C. 2006, A\&A, 451, L51

Hoshino, M., Arons, J., Gallant, Y. A., \& Langdon, A. B. 1992, ApJ, 390, 454

Ingraham, R. L. 1973, ApJ, 186, 625

Kanbach, G., Kellner, S., Schrey, F. Z., et al. 2003, in Instrument Design and Performance for Optical/Infrared Ground-based Telescopes. Edited by Iye, Masanori; Moorwood, Alan F. M. Proceedings of the SPIE, Volume 4841, pp. 82-93 (2003)., 82-93

Kaspi, V. M., Roberts, M. S. E., \& Harding, A. K. 2004, ArXiv Astrophysics e-prints

Kennel, C. F. \& Coroniti, F. V. 1984a, ApJ, 283, 694

Kennel, C. F. \& Coroniti, F. V. 1984b, ApJ, 283, 710

Khangoulian, D. V. \& Bogovalov, S. V. 2003, Astronomy Letters, 29, 495

Kirk, J. G. \& Duffy, P. 1999, Journal of Physics G Nuclear Physics, 25, 163

Kirk, J. G., Guthmann, A. W., Gallant, Y. A., \& Achterberg, A. 2000, ApJ, 542,235

Kirk, J. G. \& Skjæraasen, O. 2003, ApJ, 591, 366

Kirk, J. G., Skjæraasen, O., \& Gallant, Y. A. 2002, A\&A, 388, L29

Komissarov, S. S. 2006, MNRAS, 367, 19

Komissarov, S. S. \& Lyubarsky, Y. E. 2003, MNRAS, 344, L93

Komissarov, S. S. \& Lyubarsky, Y. E. 2004, MNRAS, 349, 779

Krause-Polstorff, J. \& Michel, F. C. 1985, A\&A, 144, 72

Kuiper, L., Hermsen, W., Cusumano, G., et al. 2001, A\&A, 378, 918

Larrabee, D. A., Lovelace, R. V. E., \& Romanova, M. M. 2003, ApJ, 586, 72

Li, Z.-Y., Chiueh, T., \& Begelman, M. C. 1992, ApJ, 394, 459

Lyubarsky, Y. 2005, Advances in Space Research, 35, 1112

Lyubarsky, Y. \& Eichler, D. 2001, ApJ, 562, 494

Lyubarsky, Y. \& Kirk, J. G. 2001, ApJ, 547, 437

Lyubarsky, Y. E. 2002, MNRAS, 329, L34

Lyubarsky, Y. E. 2003a, MNRAS, 339, 765

Lyubarsky, Y. E. 2003b, MNRAS, 345, 153

Marsden, P. L., Gillett, F. C., Jennings, R. E., et al. 1984, ApJ, 278, L29

McKinney, J. C. 2006, MNRAS, 368, L30

Melatos, A. \& Melrose, D. B. 1996, MNRAS, 279, 1168

Michel, F. C. 1969, ApJ, 158, 727

Michel, F. C. 1971, Comments on Astrophysics and Space Physics, 3, 80

Michel, F. C. 1973, ApJ, 180, L133+

Michel, F. C. 1974, ApJ, 187, 585

Michel, F. C. 1991, Theory of neutron star magnetospheres (Chicago, IL, University of Chicago Press, 1991, 533 p.)

Mori, K., Burrows, D. N., Hester, J. J., et al. 2004, ApJ, 609, 186

Ng, C.-Y. \& Romani, R. W. 2004, ApJ, 601, 479 
Ostrowski, M. \& Bednarz, J. 2002, A\&A, 394, 1141

Pacini, F. 1967, Nature, 216, 567

Pavlov, G. G., Teter, M. A., Kargaltsev, O., \& Sanwal, D. 2003, ApJ, 591, 1157

Pétri, J. 2006, in Stellar Fluid Dynamics and Numerical Simulations: From the Sun to Neutron Stars. Edited by M. Rieutord and B. Dubrulle, 355-382

Pétri, J., Heyvaerts, J., \& Bonazzola, S. 2002, A\&A, 384, 414

Pétri, J. \& Kirk, J. G. 2005, ApJ, 627, L37

Pétri, J. \& Lyubarsky, Y. 2007, submitted, 00, 000

Piddington, J. H. 1957, Australian Journal of Physics, 10, 530

Rees, M. J. \& Gunn, J. E. 1974, MNRAS, 167, 1

Reville, B., Kirk, J. G., \& Duffy, P. 2006, Plasma Physics and Controlled Fusion, 48, 1741

Romanova, M. M. \& Lovelace, R. V. E. 1992, A\&A, 262, 26

Scargle, J. D. 1969, ApJ, 156, 401

Spitkovsky, A. 2005, in AIP Conf. Proc. 801: Astrophysical Sources of High

Energy Particles and Radiation, ed. T. Bulik, B. Rudak, \& G. Madejski, 345-350

Spitkovsky, A. 2006, ApJ, 648, L51

Spitkovsky, A. \& Arons, J. 2004, ApJ, 603, 669

Timokhin, A. N. 2006, MNRAS, 368, 1055

Tomimatsu, A. 1994, Publ. Astron. Soc. Japan, 46, 123

Usov, V. V. 1975, Astrophys. Space Sci., 32, 375

van der Swaluw, E. 2003, A\&A, 404, 939

van der Swaluw, E., Achterberg, A., Gallant, Y. A., \& Tóth, G. 2001, A\&A, 380,309

Veron-Cetty, M. P. \& Woltjer, L. 1993, A\&A, 270, 370

Vlahakis, N. 2004, ApJ, 600, 324

Weisskopf, M. C., Hester, J. J., Tennant, A. F., et al. 2000, ApJ, 536, L81

Wilson, D. B. \& Rees, M. J. 1978, MNRAS, 185, 297

Zenitani, S. \& Hoshino, M. 2001, ApJ, 562, L63

Zenitani, S. \& Hoshino, M. 2005a, ApJ, 618, L111

Zenitani, S. \& Hoshino, M. 2005b, Physical Review Letters, 95, 095001 